\title{
Editorial
}

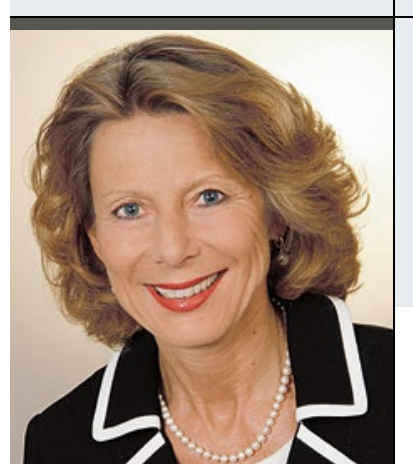

"Die Psychiatrie und Psychotherapie ist wie kaum eine andere medizinische Disziplin eng mit aktuellen politischen und gesellschaftlichen Geschehnissen verwoben."

\section{Der Mensch im Mittelpunkt: Versorgung neu denken}

edes Jahr im November trifft sich in Berlin die Fachwelt zum DGPPN-Kongress, der größten europäischen Tagung zur psychischen Gesundheit. Über 650 Einzelveranstaltungen mit rund 1.600 Referenten stehen auf dem Programm. Insgesamt werden mehr als 9.000 Wissenschaftler, Ärzte und Therapeuten erwartet.

Die Psychiatrie und Psychotherapie ist wie kaum eine andere medizinische Disziplin eng mit aktuellen politischen und gesellschaftlichen Geschehnissen verwoben. Die enorme thematische Breite des Fachgebiets spiegelt sich auch im Kongressprogramm wider, das mit einem umfassenden Überblick über die neuesten Entwicklungen in Forschung und Therapie aufwartet.

Das diesjährige Kongressmotto „Der Mensch im Mittelpunkt: Versorgung neu denken“ rückt ein zukunftsweisendes Thema in den Vordergrund. Dahinter steht die Frage, wie wir heute und in Zukunft sicherstellen können, dass psychisch erkrankte Menschen qualitativ hochwertige, wissenschaftlich fundierte und individualisierte, therapeutische Hilfen erhalten. Mit dem erstmalig eingerichteten "Schwerpunkt Hausarztpraxis“ nimmt die DGPPN in diesem Jahr einen wichtigen Aspekt der Versorgung in den Fokus. Ein besonderes Augenmerk liegt auf der ambulanten Versorgung. Dabei stellt insbesondere die stärkere Vernetzung von Klinikern und niedergelassenen Kolleginnen und Kollegen eine wesentliche Aufgabe für die Zukunft dar.

Der Kongress beschäftigt sich in diesem Jahr in einem Schwerpunkt mit der Überwindung der Fraktionierung der Versorgungsangebote sowie dem Abbau von Sektorengrenzen und der Entwicklung strukturierter Kooperationen. Innovative Versorgungskonzepte sollen vorgestellt und das Potenzial von gestuften, bedarfsgerechten, personenzentrierten Versorgungsmodellen mit nationalen und internationalen Experten diskutiert werden. In die- sem Zusammenhang gilt es auch, die Qualität und Transparenz in der Psychiatrie weiter zu verbessern. Die Umsetzung evidenzbasierter Leitlinien, aber auch ethische Fragestellungen zur Patientenautonomie oder Verteilungsgerechtigkeit von Ressourcen, stehen dabei im Zentrum der Betrachtung.

Hochaktuell ist zurzeit die Flüchtlingsproblematik, die unsere Gesellschaft im Allgemeinen, aber auch unser Fachgebiet im Speziellen vor große Aufgaben stellt. Auf dem Kongress werden wir uns intensiv mit diesen Fragen beschäftigen, etwa in der besonderen Veranstaltung „Trauma and migration - refugees and asylum seekers in mental health care“, unter anderem mit Beteiligung von Dinesh Bhugra, dem amtierenden Präsidenten der World Psychiatric Association (WPA).

Gerne weise ich Sie an dieser Stelle exemplarisch auf einige Highlights des DGPPN-Kongresses 2015 hin:

_ Im Rahmen der Kongresseröffnung wird der bekannte Medizinethiker Giovanni Maio sprechen. Maio leitet das Institut für Ethik und Geschichte der Medizin an der Albert-Ludwigs-Universität Freiburg. Seine Schriften sind ein Plädoyer für den Weg zu einer Heilkunst, die den Patienten als Menschen und nicht als „Kunden“ im Blick hat und die Gesundheit nicht als Ware verkauft. In seinem Vortrag begründet er, warum die Industrialisierung der Psychiatrie einem falschen Paradigma folgt.

_ Der amerikanische Schizophrenieforscher Jeffrey A. Lieberman gibt in einem Plenarvortrag Einblick in seine aktuellen Forschungsprojekte. Lieberman war von 2013 bis 2014 Präsident der American Psychiatric Association (APA). Seine Arbeit hat wesentliche Erkenntnisse im Bereich Entstehungsgeschichte, Pathophysiologie sowie Behandlung der Schizophrenie und damit verbundene psychotische Störungen geliefert. In seiner 


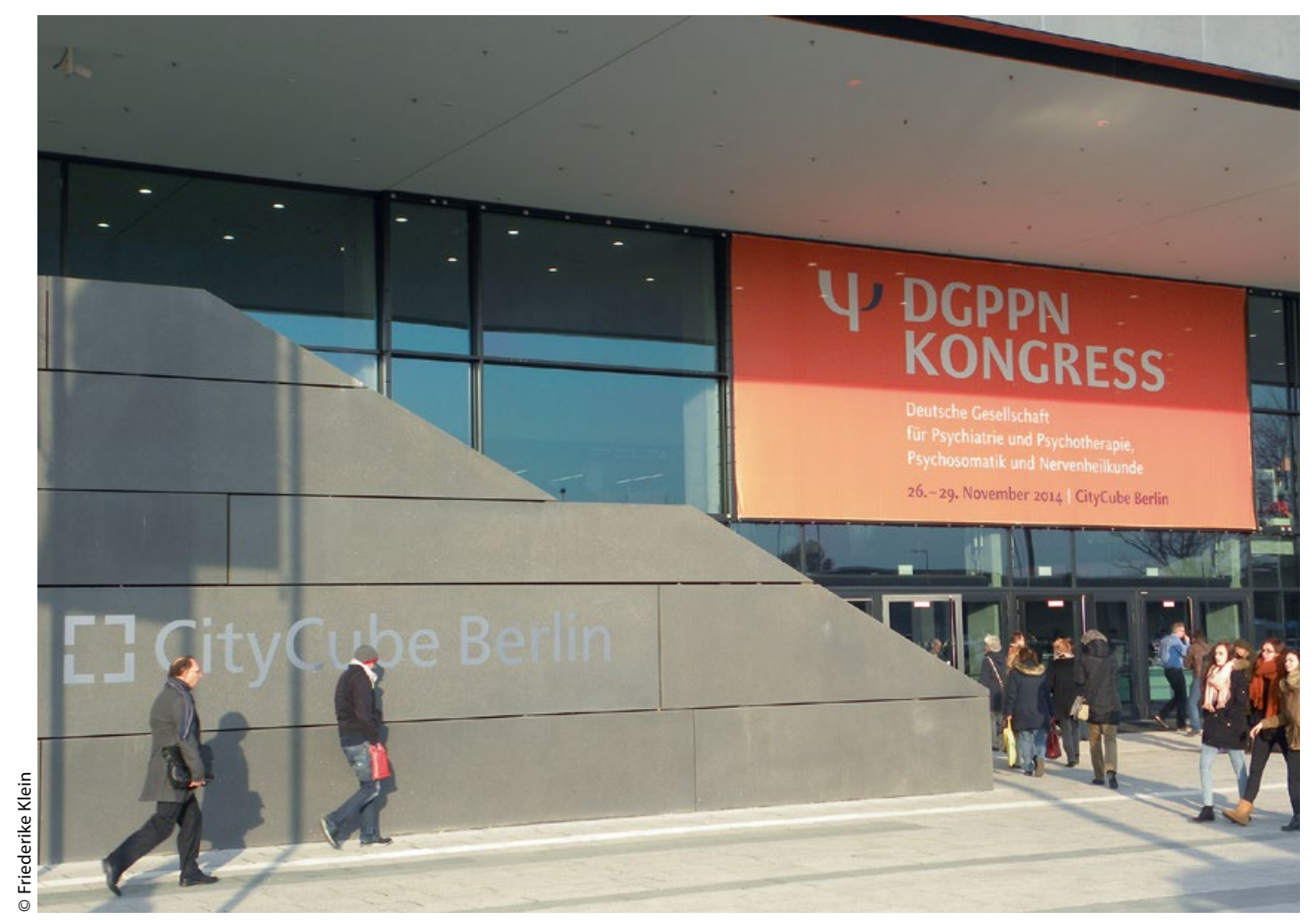

Im letzten Jahr fand der DGPPN-Kongress erstmals im Berliner CityCube statt.

Lecture wird er außerdem über „The dark past and bright future of psychiatry" sprechen.

- Jacques P. Barber vom renommierten Derner Institute of Advanced Psychological Studies, Adelphi in New York, hat neben der Behandlungsforschung auch Untersuchungen zu individuellen Kernkonflikten, Persönlichkeit und Metakognition in verschiedenen Bevölkerungsgruppen zum Beispiel bei Kindern von Holocaustüberlebenden - durchgeführt. Das Hauptinteresse seiner Forschung sind Wirkweisen von Psychotherapieverfahren, insbesondere psychodynamischer und kognitiver Therapien. Er referiert im Rahmen einer Plenarsitzung über „The present and future of psychodynamic therapy research".

_-In diesem Jahr wird die Konsultationsfassung der vollständig überarbeiteten „S3-Leitlinie und Nationale Versorgungsleitlinie (NVL) Unipolare Depression“ fertiggestellt. Sie will die Erkennung, Diagnostik und Behandlung von Depressionen in Deutschland nachhaltig verbessern. Die Leitlinie formuliert auf über 260 Seiten mehr als 130 Schlüsselempfehlungen zu Diagnostik und Therapie sowie zu prioritären Versorgungsproblemen. Initiiert und koordiniert hat die Leitlinie die DGPPN. Im dazugehörigen Hauptsymposium „Nationale Versorgungsleitlinie Unipolare De- pression" am 25. November werden die konkreten Inhalte der Leitlinie vorgestellt und diskutiert.

_Ein gesellschaftlich brisantes Thema greifen wir im Diskussionsforum „Radikalisierung - wenn Menschen extrem werden“ auf. Dieses geht der Frage nach, welche Voraussetzungen zur Entstehung von Radikalisierungsprozessen und Extremüberzeugungen beitragen. Es wird interdisziplinär diskutiert, ob ein psychopathologischer Zugang zu dem Thema hilft, das Phänomen besser zu verstehen und wirksame Handlungs- und Präventionsmaßnahmen zu entwickeln.

Ich hoffe, Sie vom 25. bis 28. November $2015 \mathrm{im}$ CityCube Berlin begrüßen zu dürfen und freue mich auf Impulse und Anregungen in der gemeinsamen Diskussion.

Mit herzlichen Grüßen,

Ihre

Iris Hauth

Dr. med. Iris Hauth

Präsidentin der DGPPN, Alexianer St. JosephKrankenhaus Berlin-Weißensee, Zentrum für Neurologie, Psychiatrie, Psychotherapie Gartenstr. 1, 13088 Berlin 